\title{
35 CHEMOKINE-DRIVEN SPATIAL ORGANIZATION OF IMMUNE CELL MICROAGGREGATES MARKS OROPHARYNGEAL SQUAMOUS CELL CARCINOMAS CONTAINING TUMOR-SPECIFIC T CELLS
}

\footnotetext{
${ }^{1}$ Ziena Abdulrahman*, 'Saskia Santegoets, ${ }^{2}$ Gregor Sturm, ${ }^{3}$ Pornpimol Charoentong, ${ }^{4}$ Marieke ljsselsteijn, ${ }^{4}$ Antonios Somarakis, ${ }^{4}$ Thomas Höllt, ${ }^{2}$ Francesca Finotello, ${ }^{2}$ Zlatko Trajanoski, ${ }^{4}$ Sylvia van Egmond, ${ }^{5}$ Dana Mustafa, ${ }^{1}$ Marij Welters, ${ }^{4}$ Noel de Miranda, ${ }^{1}$ Sjoerd van der Burg. 'Leiden University Medical Center, Oncode Institute, Leiden, Netherlands; ${ }^{2}$ Medical University of Innsbruck, Innsbruck, Austria; ${ }^{3}$ University Hospital Heidelberg, Heidelberg, Germany; ${ }^{4}$ Leiden University Medical Center, Leiden, Netherlands; ${ }^{5}$ Erasmus University Medical Center, Rotterdam, Netherlands
}

Background Oropharyngeal squamous cell carcinoma (OPSCC) is the most prevalent type of head and neck cancer. The survival of patients with OPSCC is tightly linked to the intratumoral presence of tumor-specific CD4+ and CD8 $+\mathrm{T}$ cells. Yet, immunotherapy is currently far from effective in OPSCC partly due to our limited understanding of its immune microenvironment.

Methods Here a multi-modal, high-dimensional approach was used to dissect the immune landscape in a unique cohort of pre-therapy OPSCC patient samples $(n=20)$ in which intratumoral tumor-specific $\mathrm{T}$ cells were either detected (immune response positive, IR+) or not (IR-). This included imaging mass cytometry (Hyperion) for high-dimensional phenotyping, spatial localization and interaction analyses of the cells in the tumor mircoenvironment with our newly developed imaging processing pipeline employing machine learning, Nanostring PanCancer IO360 panel analysis of immune signaling pathways, and combined single-cell gene expression profiling and $T$ cell receptor sequencing (scRNAseq) to characterize the transcriptional states of clonally expanded tumor-infiltrating $\mathrm{T}$ cells.

Results Immune cell infiltration in IR + tumors is stronger and highly coordinated, with a distinct spatial phenotypic signature characterized by microaggregates of tumor-resident (CD103+) CD8 + and CD4+ T cells and dendritic cells within the tumor cell beds, which retained after permutation based correction for differences in cell frequencies. Furthermore, the increased expression of CXCL12 and LTB produced by CD4+ T cells, both involved in the spatial organization of immune cell infiltration, and the clonal expansion of $\mathrm{CD} 8+\mathrm{T}$ cells producing the DC-attracting chemokines CCL4 or XCL1 in IR+ OPSCC, indicate that tumor-reactive $\mathrm{T}$ cells act as a positive feedback loop in the formation of these aggregates. The impact of these chemokines on local immunity and clinical outcome was confirmed in an independent TCGA OPSCC cohort. In contrast, the IR- OPSCC signature comprised spatial interactions between lymphocytes and different subpopulations of immunosuppressive myeloid cells.

Conclusions Our study reveals that the chemokine-driven spatial immune signature of OPSCC has strong potential as a prognostic and predictive biomarker. While the immune signature of IR+ OPSCC suggests potential benefit from neoadjuvant immunotherapeutic approaches to limit the side effects of current radio(chemo)therapy, that of IR- OPSCC calls for strategies focused on stimulating $\mathrm{T}$ cells and counteracting immune suppressive mechanisms.

http://dx.doi.org/10.1136/jitc-2021-SITC2021.035 